\title{
HARDY'S UNCERTAINTY PRINCIPLE AND UNIQUE CONTINUATION PROPERTY FOR STOCHASTIC HEAT EQUATIONS
}

\author{
Aingeru FernándeZ-Bertolin ${ }^{1}$ And Jie Zhong ${ }^{2}$
}

\begin{abstract}
The goal of this paper is to prove a qualitative unique continuation property at two points in time for a stochastic heat equation with a randomly perturbed potential, which can be considered as a variant of Hardy's uncertainty principle for stochastic heat evolutions.
\end{abstract}

1991 Mathematics Subject Classification. 35B05, 35B60, 60H15.

The dates will be set by the publisher.

\section{INTRODUCTION}

It is well known that the unique continuation property has extensive applications in control theory of partial differential equations, especially observability for the system; see [31] for details, or 20, 27, 30 for the stochastic case. Aforementioned works deal with uniqueness in domains, that is, under certain conditions, if a solution is zero in a subset, then the solution is identically zero. Recently, there is a series of studies [5,7-12] on uniqueness of two time points, where the goal is to understand sufficient conditions for the solution at two different times so that the solution vanishes. The methodology involved in their project is very robust, as it can be seen in extensions of their results from heat equations to the magnetic Schrödinger equation 2, 4 and more recently to the discrete Schrödinger equation 3, 13, 17]. We refer to 22 for an extension of these uniqueness results to more general parabolic equations. It is also worth mentioning that 26] studied the relation of uncertainty principle and observability in control theory.

The motivation of proving such unique continuation properties for solutions of heat or Schrödinger equations knowing the behavior of the solution at two different times comes from the very famous result of G. H. Hardy [16 or 25, page 131], concerning the decay of a function $f$ and its Fourier transform

$$
\hat{f}(\xi)=(2 \pi)^{-\frac{n}{2}} \int_{\mathbb{R}^{n}} e^{-i \xi \cdot x} f(x) d x .
$$

Keywords and phrases: Hardy uncertainty principle, unique continuation, stochastic heat equation

1 Universidad del País Vasco / Euskal Herriko Unibertsitatea, Dpto Matemáticas, Barrio Sarriena s/n 48940 Leioa, Spain. e-mail: aingeru.fernandez@ehu.eus

2 Department of Mathematics, California State University Los Angeles, 5151 State University Drive, Los Angeles, CA 90032, USA. e-mail: jiezhongmath@gmail.com

(c) EDP Sciences, SMAI 1999 
Under this definition of the Fourier transform, Hardy proves:

If $f(x)=O\left(e^{-|x|^{2} / \beta^{2}}\right), \hat{f}(\xi)=O\left(e^{-4|\xi|^{2} / \alpha^{2}}\right)$ and $\alpha \beta<4$, then $f \equiv 0$. Also, if $\alpha \beta=4$, $f$ is a constant multiple of $e^{-|x|^{2} / \beta^{2}}$.

Since its original formulation, the Hardy uncertainty principle has been extended to more general settings. For instance, we have the following $L^{2}$-version of the uncertainty principle 24 :

$$
e^{|x|^{2} / \beta^{2}} f, e^{4|\xi|^{2} / \alpha^{2}} \hat{f} \in L^{2}\left(\mathbb{R}^{n}\right) \text { and } \alpha \beta \leq 4 \Longrightarrow f \equiv 0 .
$$

Moreover, thanks to the expression of solutions of free Schrödinger and heat equations, it is possible to rewrite the Hardy uncertainty principle in terms of solutions of these equations. Since we are concerned with the heat equation, in this case it is known that

$$
f, e^{|x|^{2} / \delta^{2}} e^{\Delta} f \in L^{2}\left(\mathbb{R}^{n}\right) \text { for some } \delta \leq 2 \Longrightarrow f \equiv 0 .
$$

Due to logarithmic convexity properties of solutions with fast decay properties at two different times, the authors extend in 7, 10 this dynamic Hardy uncertainty principle to solutions of the equation $\partial_{t} u=\Delta u+V u$, where the potential $V$ is bounded, using only real variable techniques, whereas the previous known proofs of the Hardy uncertainty principle, up to the endpoint case, were based on complex analysis arguments. In the preliminary non-sharp version of the result in [10], they prove first that a solution with Gaussian decay at time $t_{0}=0$ and $t_{1}=1$ preserves this decay at any time in between, and, furthermore, in the open interval $(0,1)$ the solution exhibits better decay properties. Combining this result with a Carleman estimate, they are able to conclude uniqueness for solutions with a non-sharp rate of decay. Every step of the proof follows a formal approach that is justified at the end of the proof, which represents a considerable technical difficulty.

In this paper, we provide a uniqueness result of two time points for the following stochastic heat equation with random potential and multiplicative random noise:

$$
\left\{\begin{array}{l}
d u=(\Delta u+V(t, x) u) d t+G(t, x) u d W(t), \quad(t, x) \in(0,1] \times \mathbb{R}^{n}, \\
u(0)=u_{0},
\end{array}\right.
$$

which formally can be viewed as a heat evolution with a randomly perturbed potential $V+G \dot{W}$. Our aim is to explore sufficient conditions for the solution $u$ of equation (1.1), the potential $V$, the noise $G$ and the behavior of the solution at two different times $t_{0}=0$ and $t_{1}=1$, in order to guarantee that $u \equiv 0$. To the best of our knowledge, this is the first work to study the stochastic counterpart in this topic.

It is reasonable to think that in the presence of a noise term, the statement will not change, at least for small noises. We see in this paper that the approach introduced in [10] can be formally adapted to our setting to extend the Hardy uncertainty principle, however, the justification procedure is much more challenging. As a byproduct, a new interior regularity for the stochastic heat equation, with a quadratic exponential weight is provided, and the result itself is also interesting.

Before we state our main theorem, we need to assume the following hypothesis on the potential $V$ and the noise $G$ in equation 1.1 .

Assumption 1.1. The measurable functions $V$ and $G: \Omega \times[0,1] \times \mathbb{R}^{n} \rightarrow \mathbb{R}$ satisfy the following conditions:

(1) $V, G$ and $\nabla G$ are bounded on $\Omega \times[0,1] \times \mathbb{R}^{n}$; 
(2) Given $\gamma>0$, there exists $\varepsilon>0$ such that

$$
\begin{aligned}
\sup _{\substack{t \in[0,1] \\
\omega \in \Omega}}|G(\omega, t, x)| \leq \frac{\sqrt{\gamma}}{(\sqrt{1+4 \gamma})^{1-\varepsilon}}|x|^{-\varepsilon}, \quad \text { for }|x| \geq \frac{\max \{\gamma, 2\}}{\sqrt{1+4 \gamma}} ; \\
\text { (3) } \lim _{L \rightarrow \infty} \sup _{\substack{t \in[0,1],|x|>L \\
\omega \in \Omega}}|V(\omega, t, x)|=\lim _{L \rightarrow \infty} \sup _{\substack{t \in[0,1],|x|>L \\
\omega \in \Omega}}|\nabla G(\omega, t, x)|=0 .
\end{aligned}
$$

Notice that the potential $V$ considered in the deterministic case is bounded whereas in the stochastic setting we require it to slightly decay at infinity. If we only carry out the formal arguments, we do not need neither the potential nor the noise to decay, but we cannot forget that formal computations could lead to misleading conclusions, as for example, it happens in the deterministic case for the Schrödinger equation (see Section 6 in 10 ). As we point out in Remark 3.5 we could relax condition (3) and assume that both $V$ and $\nabla G$ are bounded, with small enough bounds when $|x|$ is large. Since this would also be a technical assumption, for the sake of simplicity we use condition (3) instead.

Condition (2) has a different nature. In the deterministic case, the approach to conclude uniqueness has two steps. In the first step (Lemma 3 in 10]) the authors prove a logarithmic convexity estimate to see that the solution has Gaussian decay at all times. Then, the second step (Lemma 4 in [10]) consists of proving that in the interior of $[0,1]$, both $x u$ and $\nabla u$ have also Gaussian decay. However, in the stochastic case, we have to show first the interior regularity properties for the gradient of the solution, as explained in Remark 3.6. Roughly speaking, we first prove an analogous version of Lemma 4 to conclude an analogous version of Lemma 3 in [10, which is done in Lemma 3.4 and is where condition (2) appears.

Once we prove these interior properties, we can follow two different approaches. On the one hand, we could prove a logarithmic convexity estimate analogous to Lemma 3 in 10 and then conclude uniqueness from this logarithmic convexity result. On the other hand, we can combine the interior decay properties in Lemma 3.4 with a Carleman estimate to get uniqueness. In this paper, we follow the second approach, since, even though one can think that both approaches could be equivalent, it allows to consider a wider class of noises. As in deterministic equations, Carleman estimates are extensively used in stochastic PDEs, for example, inverse problems or to prove uniqueness (see [30], 21], 28]), and to show observability or controllability (see [1, [29, [15], 19]). In this work, we follow the idea from [18], where the author applied Carleman estimates to study inverse problems.

Let us introduce some basic notations.

Let $\mathbb{F}=\left(\Omega, \mathcal{F},\left\{\mathcal{F}_{t}\right\}_{t \geq 0}, \mathbb{P}\right)$ be a stochastic basis with usual conditions. On $\mathbb{F}$, we define a standard scalar Wiener process $W=\{W(t)\}_{t \geq 0}$. We assume that the filtration $\left\{\mathcal{F}_{t}\right\}_{t \geq 0}$ is generated by $W$.

Given a Hilbert space $H$, we denote by $L_{\mathcal{F}}^{2}([0,1] ; H)$ the Banach space consisting of all $H$-valued $\left\{\mathcal{F}_{t}\right\}_{t \geq 0}$-adapted processes $X$ such that the square of the canonical norm $\mathbb{E} \int_{0}^{1}\|X(t)\|_{H}^{2} d t<\infty$; and denote by $C_{\mathcal{F}}([0,1] ; H)$ the Banach space consisting of all $H$-valued $\left\{\mathcal{F}_{t}\right\}_{t \geq 0}$-adapted continuous processes $X$ such that the square of the canonical norm $\mathbb{E} \sup _{0 \leq t \leq 1}\|X(t)\|_{H}^{2}<\infty$.

We denote by $(\cdot, \cdot)$ the inner product in $L^{2}\left(\mathbb{R}^{n}\right)$ and denote by $\|\cdot\|$ the norm induced by $(\cdot, \cdot)$.

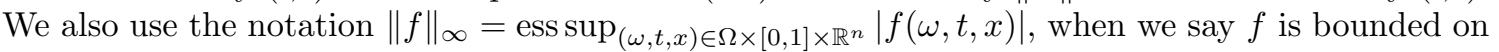
$\Omega \times[0,1] \times \mathbb{R}^{n}$. 
Definition 1.2. We say $u$ is a solution of equation 1.1 if $u$ is in the space of $C_{\mathcal{F}}\left([0,1] ; L^{2}\left(\mathbb{R}^{n}\right)\right) \bigcap L_{\mathcal{F}}^{2}\left([0,1] ; H^{1}\left(\mathbb{R}^{n}\right)\right)$ such that for all $\varphi \in C_{c}^{\infty}\left(\mathbb{R}^{n}\right)$ and all $t \in[0,1]$ we have

$$
(u(t), \varphi)=\left(u_{0}, \varphi\right)+\int_{0}^{t}(\nabla u(s), \nabla \varphi) d s+\int_{0}^{t}(V u(s), \varphi) d s+\int_{0}^{t}(G u(s), \varphi) d W(s), \mathbb{P} \text {-a.s. }
$$

The following is our main result.

Theorem 1.3. Suppose $u$ is a solution of equation 1.1, and assume that

$$
\mathbb{E}\left\|u_{0}\right\|^{2}<\infty, \quad \mathbb{E}\left\|e^{|x|^{2} / \delta^{2}} u(1)\right\|^{2}<\infty
$$

for some $0<\delta<1$. Then we have $u \equiv 0$ in $[0,1] \times \mathbb{R}^{n}$, $\mathbb{P}$-a.s. if either $V$ is bounded in $[0,1] \times \mathbb{R}^{n}$ and $G=G(t)$ is a bounded function in $[0,1]$, or Assumption 1.1 holds and

$$
\|G\|_{\infty}^{2}<\frac{4 \gamma}{1+4 \gamma}
$$

where $\gamma=1 /(2 \delta)$.

This result is not likely to be sharp, compared to the deterministic equation, but a combination of it with the procedure developed in 7. will probably start a self-improvement argument. On the other hand, it is reasonable to claim that a similar result holds for Schrödinger evolutions. We are currently working on both projects.

The first part of our main result deals with a deterministic noise $G=G(t)$, independent of the space variable $x$. In this case, it is easy to see from some obvious transform that the deterministic result still holds. This can also be verified through the proof of a logarithmic convexity result analogous to Lemma 3 in [10] (see Remark 3.2). In particular, this implies that the deterministic result $(G \equiv 0)$ is trivially included in our statement, and, therefore, in the sequel we only focus on the case that $G$ is random and depends on $x$.

Furthermore, if we consider $V$ and $G$ as deterministic functions, one can apply the deterministic result proved in 7 to the function $\mathbb{E} u$, and conclude that the following statement holds true for solutions of 1.1 with bounded deterministic potential $V$ and deterministic noise $G$ :

$$
\mathbb{E}\left\|u_{0}\right\|^{2}, \mathbb{E}\left\|e^{|x|^{2} / \delta^{2}} u(1)\right\|^{2}<\infty \text { for some } \delta<2 \Longrightarrow \mathbb{E} u \equiv 0 .
$$

Theorem 1.3 shows that if $0<\delta<1$ not only is our solution zero on expectation, but it is zero $\mathbb{P}$-almost surely. Moreover, it also extends the result to stochastic processes $V$ and $G$.

The rest of the paper is organized as follows: in Section 2, we provide necessary estimates, especially the interior regularity for the decay of the solution. In Section 3, we first study formally the Gaussian decay of the solution at the interior, and then focus on rigorous justifications. Section 4 is devoted to the proof of our main result Theorem 1.3 .

\section{Preliminary estimates}

In this section, we start with the energy estimate for the solution $u$ of equation 1.1 , multiplied by a quadratic exponential weight function. 
Lemma 2.1 (Energy estimate). Suppose $u$ is a solution of equation (1.1). Then there is a constant $C>0$ such that

$$
\mathbb{E} \sup _{0 \leq t \leq 1}\left\|e^{\phi_{\gamma}(t)|x|^{2}} u(t)\right\|^{2} \leq e^{C M_{G, V}} \mathbb{E}\left\|e^{\gamma|x|^{2}} u_{0}\right\|^{2}
$$

where $\gamma \geq 0, \phi_{\gamma}(t)=\gamma /(1+4 \gamma t)$, and $M_{G, V}=\|G\|_{\infty}^{2}+2\|V\|_{\infty}$.

Proof. Formally, let $v=e^{\varphi(t, x)} u$ with $\varphi(t, x)=\phi_{\gamma}(t)|x|^{2}$, then by Itô's formula we have

$$
d v=\partial_{t} \varphi v d t+\left[-\Delta \varphi v-2 \nabla \varphi \cdot \nabla v+\Delta v+|\nabla \varphi|^{2} v+V v\right] d t+G v d W(t) .
$$

Applying Itô's formula for $\|v\|^{2}$ and integration by parts yield

$$
\begin{aligned}
\|v(t)\|^{2}+2 \int_{0}^{t}\|\nabla v\|^{2} d s=\|v(0)\|^{2}+ & 2 \int_{0}^{t} \int_{\mathbb{R}^{n}}\left(|\nabla \varphi|^{2}+\partial_{t} \varphi\right) v^{2} d x d s \\
& +\int_{0}^{t}\|G v(s)\|^{2} d s+2 \int_{0}^{t}(v, V v) d s+2 \int_{0}^{t}(v, G v) d W(s) .
\end{aligned}
$$

It is clear that

$$
|\nabla \varphi|^{2}+\partial_{t} \varphi=0
$$

and thus we obtain

$$
\begin{aligned}
\|v(t)\|^{2}+2 \int_{0}^{t}\|\nabla v\|^{2} d s=\|v(0)\|^{2} & \\
& +\int_{0}^{t}\|G v(s)\|^{2} d s+2 \int_{0}^{t}(v, V v) d s+2 \int_{0}^{t}(G v, v) d W(s) .
\end{aligned}
$$

Taking expectation on both sides and getting rid of the gradient term, we have

$$
\begin{aligned}
\mathbb{E}\|v(t)\|^{2} & \leq \mathbb{E}\|v(0)\|^{2}+\mathbb{E} \int_{0}^{t}\|G v(s)\|^{2} d s+2 \mathbb{E} \int_{0}^{t}|(v, V v)| d s \\
& \leq \mathbb{E}\|v(0)\|^{2}+\|G\|_{\infty}^{2} \mathbb{E} \int_{0}^{t}\|v(s)\|^{2} d s+2\|V\|_{\infty} \mathbb{E} \int_{0}^{t}\|v(s)\|^{2} d s \\
& =\mathbb{E}\|v(0)\|^{2}+M_{G, V} \mathbb{E} \int_{0}^{t}\|v(s)\|^{2} d s .
\end{aligned}
$$

It follows from Gronwall's inequality that

$$
\mathbb{E}\|v(t)\|^{2} \leq e^{M_{G, V}} \mathbb{E}\|v(0)\|^{2},
$$

which also implies that $\mathbb{E} \int_{0}^{1}\|v(t)\|^{2} d t \leq e^{M_{G, V}} \mathbb{E}\|v(0)\|^{2}$. 
Now we go back to the equality (2.1), and use the Burkholder-Davis-Gundy's inequality with $p=1$ to estimate $\mathbb{E} \sup _{0 \leq t \leq 1}\|v(t)\|^{2}$ as follows:

$$
\begin{aligned}
\mathbb{E} \sup _{0 \leq t \leq 1}\|v(t)\|^{2} \leq \mathbb{E}\|v(0)\|^{2}+\|G\|_{\infty}^{2} \mathbb{E} \int_{0}^{1}\|v(s)\|^{2} d s+2\|V\|_{\infty} \int_{0}^{1}\|v(s)\|^{2} d s \\
\quad+2 \mathbb{E} \sup _{0 \leq t \leq 1}\left|\int_{0}^{t}(G v, v) d W(s)\right| \\
\leq \mathbb{E}\|v(0)\|^{2}+M_{G, V} \mathbb{E} \int_{0}^{1}\|v(s)\|^{2} d s+C \mathbb{E}\left(\int_{0}^{1}|(G v, v)|^{2} d s\right)^{1 / 2} \\
\leq \mathbb{E}\|v(0)\|^{2}+M_{G, V} \mathbb{E} \int_{0}^{1}\|v(s)\|^{2} d s+\frac{1}{2} \mathbb{E} \sup _{0 \leq t \leq 1}\|v(t)\|^{2} \\
+C\|G\|_{\infty}^{2} \mathbb{E} \int_{0}^{1}\|v(s)\|^{2} d s .
\end{aligned}
$$

Therefore,

$$
\begin{aligned}
\mathbb{E} \sup _{0 \leq t \leq 1}\|v(t)\|^{2} & \leq 2 \mathbb{E}\|v(0)\|^{2}+2\left(M_{G, V}+C\|G\|_{\infty}^{2}\right) \mathbb{E} \int_{0}^{1}\|v(t)\|^{2} d t \\
& \leq 2 \mathbb{E}\|v(0)\|^{2}+2\left(M_{G, V}+C\|G\|_{\infty}^{2}\right) e^{M_{G}, V} \mathbb{E}\|v(0)\|^{2} \\
& \leq e^{C M_{G, V}} \mathbb{E}\|v(0)\|^{2} .
\end{aligned}
$$

To justify the integration by parts and calculations carried out above, we use the same truncation and mollification as in [10, Lemma 1], which completes the proof.

Interior regularity (or smoothing property) for deterministic parabolic equations is standard and well known, i.e., the solution becomes smooth for any $t>0$, even though the initial data may be singular. Similar but more subtle result for stochastic equations can be proved, see for example [14]. However, in the rest of this section, we will show the interior regularity for the solution $u$ of stochastic equation (1.1), with a quadratic exponential weight, which serves as an important tool for the rigorous justifications in the later sections. The result itself is also interesting and new in this stochastic context.

Let $\gamma \geq 0$. Fix $0<a<1$, let $\zeta_{a}$ be a positive function in $C_{c}^{\infty}(\mathbb{R})$ such that

$$
\zeta_{a}(r)= \begin{cases}0, & r \leq \max \{\gamma, 2\}-1 \\ 2 r^{-a}, & r \geq \max \{\gamma, 2\}\end{cases}
$$

We define $\varphi_{a}$ as a radial function in $\mathbb{R}^{n}$, i.e., $\varphi_{a}(x)=\varphi_{a}(|x|)$ satisfying

$$
\left\{\begin{array}{l}
\varphi_{a}^{\prime \prime}(r)-\varphi_{a}^{\prime}(r) / r=-a \zeta_{a}(r) \\
\varphi_{a}^{\prime}(0)=0=\lim _{r \rightarrow \infty} \varphi_{a}^{\prime \prime}(r)
\end{array}\right.
$$


Notice that $\varphi_{a}^{\prime}(r)=\operatorname{ar} \int_{r}^{\infty} \zeta_{a}(s) / s d s$. This allows us to choose $\varphi_{a}$ such that

$$
\varphi_{a}(r)= \begin{cases}\left(2 r^{2-a}-a\right) /(2-a), & \text { if } r \geq \max \{\gamma, 2\} \\ (1+O(a)) r^{2}, & \text { if } 0 \leq r \leq \max \{\gamma, 2\}\end{cases}
$$

Lemma 2.2 (Interior regularity). Assume $u$ is a solution of equation (1.1), and $V, G$ and $\nabla G$ are bounded in $\Omega \times[0,1] \times \mathbb{R}^{n}$. Then for any $\varepsilon>0$ we have

$$
\sup _{\varepsilon \leq t \leq 1} \mathbb{E}\left\|e^{\gamma \varphi_{a}(x)} \nabla u(t)\right\|^{2}+\mathbb{E} \int_{\varepsilon}^{1}\left\|e^{\gamma \varphi_{a}(x)} \mathcal{D}^{2} u(t)\right\|^{2} d t<\infty
$$

where $\varphi_{a}$ is defined as in 2.2 and 2.3 .

Proof. Define $\psi(t, y)=\eta(t) \theta(y) \in C_{0}^{\infty}\left([0,1] \times \mathbb{R}^{n}\right)$ with

$$
\eta(t)=\left\{\begin{array}{ll}
0, & t \in[0, \epsilon / 4], \\
1, & t \in[\varepsilon / 2,1],
\end{array} \quad \theta(y)= \begin{cases}1, & y \in B_{1}(x), \\
0, & y \notin B_{2}(x),\end{cases}\right.
$$

for $x$ such that $|x| \geq N$, where $B_{r}(x)$ is a ball centered at $x$ with radius $r$.

Since $d(\psi u)=\partial_{t} \psi u d t+\psi d u$, it follows from Itô's formula applied to $\|\psi u\|^{2}$ that

$$
d\|\psi u\|^{2}=2(\psi u, d(\psi u))+\|G \psi u\|^{2} d t
$$

or

$$
\begin{aligned}
\frac{1}{2}\|\psi(t) u(t)\|^{2} & =\int_{0}^{t} \int_{\mathbb{R}^{n}} \psi(s, y) \partial_{s} \psi(s, y) u^{2}(s, y) d y d s \\
& +\int_{0}^{t} \int_{\mathbb{R}^{n}} \psi^{2}(s, y) u(s, y) \Delta u(s, y) d y d s+\int_{0}^{1} \int_{\mathbb{R}^{n}} V(s, y) \psi^{2}(s, y) u^{2}(s, y) d y d s \\
& +\int_{0}^{t} \int_{\mathbb{R}^{n}} G(s, y) \psi^{2}(s, y) u^{2}(s, y) d y d W(s)+\frac{1}{2} \int_{0}^{t} \int_{\mathbb{R}^{n}} G^{2}(s, y) \psi^{2}(s, y) u^{2}(s, y) d y d s
\end{aligned}
$$

where we have used the fact that $\psi(0)=0$. Then by observing that

$$
\begin{aligned}
\int_{\mathbb{R}^{n}} \psi^{2}(t, y) u(t, y) \Delta u(t, y) d y & = \\
& -\int_{\mathbb{R}^{n}} \psi^{2}(t, y)|\nabla u(t, y)|^{2} d y-2 \int_{\mathbb{R}^{2}} \nabla \psi(t, y) \cdot \nabla u(t, y) \psi(t, y) u(t, y) d y
\end{aligned}
$$


and taking expectation we obtain

$$
\begin{aligned}
& \frac{1}{2} \mathbb{E}\|\psi(t) u(t)\|^{2}+\mathbb{E} \int_{0}^{t}\|\psi(s) \nabla u(s)\|^{2} d s \\
&= \mathbb{E} \int_{0}^{t} \int_{\mathbb{R}^{n}} \psi(s, y) \partial_{s} \psi(s, y) u^{2}(s, y) d y d s-2 \mathbb{E} \int_{0}^{t} \int_{\mathbb{R}^{n}} \nabla \psi(s, y) \cdot \nabla u(s, y) \psi(s, y) u(s, y) d y d s \\
& \quad+\mathbb{E} \int_{0}^{t} \int_{\mathbb{R}^{n}} V(s, y) \psi^{2}(s, y) u^{2}(s, y) d y d s+\frac{1}{2} \mathbb{E} \int_{0}^{t}\|G(s) \psi(s) u(s)\|^{2} d s .
\end{aligned}
$$

After using Cauchy-Schwarz inequality in the second integral on the right hand side of the previous equality, we have that there is a constant $C$ depending on $\|G\|_{\infty}$ and $\|V\|_{\infty}$ such that

$$
\sup _{\varepsilon \leq t \leq 1} \mathbb{E} \int_{y \in B_{1}(x)} u^{2}(t, y) d y+\mathbb{E} \int_{\varepsilon / 2}^{1} \int_{y \in B_{1}(x)}|\nabla u(t, y)|^{2} d y d t \leq \frac{C}{\varepsilon} \mathbb{E} \int_{\varepsilon / 4}^{1} \int_{y \in B_{2}(x)} u^{2}(t, y) d y d t .
$$

Next, let us differentiate the equation satisfied by $u$ with respect to a variable $x_{i}$ and we obtain

$$
d u_{i}=\left(\Delta u_{i}+(V u)_{i}\right) d t+(G u)_{i} d W(t)
$$

where $u_{i}=\partial_{x_{i}} u(t, x)$, and similarly for $(V u)_{i}$ and $(G u)_{i}$. Repeating the computations as before with $\psi(t, y)=\eta(t) \theta(y)$, where

$$
\eta(t)=\left\{\begin{array}{ll}
0, & t \in[0, \epsilon / 2], \\
1, & t \in[\varepsilon, 1],
\end{array} \quad \theta(y)= \begin{cases}1, & y \in B_{1 / 2}(x) \\
0, & y \notin B_{1}(x)\end{cases}\right.
$$

we get

$$
\begin{aligned}
& \sup _{\varepsilon \leq t \leq 1} \mathbb{E} \int_{y \in B_{1 / 2}(x)} u_{i}^{2}(t, y) d y+\mathbb{E} \int_{\varepsilon}^{1} \int_{y \in B_{1 / 2}(x)}\left|\nabla u_{i}(t, y)\right|^{2} d y d t \\
\leq & \frac{C}{\varepsilon} \mathbb{E} \int_{\varepsilon / 2}^{1} \int_{y \in B_{1}(x)} u(t, y)^{2} d y d t+C \mathbb{E} \int_{\varepsilon / 2}^{1} \int_{y \in B_{1}(x)}|\nabla u(t, y)|^{2} d y d t .
\end{aligned}
$$

Thus, it follows from 2.5 that

$$
\begin{aligned}
& \sup _{\varepsilon \leq t \leq 1} \mathbb{E} \int_{y \in B_{1 / 2}(x)}|\nabla u(t, y)|^{2} d y+\mathbb{E} \int_{\varepsilon}^{1} \int_{y \in B_{1 / 2}(x)}\left|\mathcal{D}^{2} u(t, y)\right|^{2} d y d t \\
\leq & \frac{C}{\varepsilon} \mathbb{E} \int_{\varepsilon / 4}^{1} \int_{y \in B_{2}(x)} u(t, y)^{2} d y d t,
\end{aligned}
$$

by summing in $i=1,2, \cdots, n$.

For $y \in B_{2}(x)$, and $|x| \geq N$ with $N$ sufficiently large, there is $\nu>0$ such that

$$
(1-\nu) \varphi(y) \leq(1-\nu / 2) \varphi(x) \leq \varphi(y)
$$

where

$$
\varphi(x)=|x|^{2} /(1+4 \gamma)
$$


Therefore,

$$
\begin{aligned}
& \sup _{\varepsilon \leq t \leq 1} \mathbb{E} \int_{y \in B_{1 / 2}(x)} e^{2(1-\nu) \gamma \varphi(y)}|\nabla u(t, y)|^{2} d y+\mathbb{E} \int_{\varepsilon}^{1} \int_{y \in B_{1 / 2}(x)} e^{2(1-\nu) \gamma \varphi(y)}\left|\mathcal{D}^{2} u(t, y)\right|^{2} d y d t \\
\leq & \frac{C}{\varepsilon} \mathbb{E} \int_{\varepsilon / 4}^{1} e^{2(1-\nu / 2) \gamma \varphi(x)} \int_{y \in B_{2}(x)} u^{2}(t, y) d y d t \\
\leq & \frac{C}{\varepsilon} \mathbb{E} \int_{\varepsilon / 4}^{1} \int_{y \in B_{2}(x)} e^{2 \gamma \varphi(y)} u^{2}(t, y) d y d t .
\end{aligned}
$$

Now by means of a covering lemma, see for example [6, Theorem 1.1], we can find a sequence $\left\{x_{j}\right\}$ with $\sup _{j}\left|x_{j}\right| \geq N$ such that $\{|y| \geq N\} \subset \bigcup_{j} B_{1 / 2}\left(x_{j}\right)$ and $\sum_{j} \chi_{B_{2}\left(x_{j}\right)} \leq C(n)$. Summing in $j$, we conclude from (2.6) and 2.8 that

$$
\begin{aligned}
& \sup _{\varepsilon \leq t \leq 1} \mathbb{E} \int_{|y| \geq N}|\nabla u(t, y)|^{2} d y+\mathbb{E} \int_{\varepsilon}^{1} \int_{|y| \geq N}\left|\mathcal{D}^{2} u(t, y)\right|^{2} d y d t \\
\leq & \frac{C}{\varepsilon} \mathbb{E} \int_{\varepsilon / 4}^{1} \int_{\mathbb{R}^{n}} u^{2}(t, y) d y d t<\infty
\end{aligned}
$$

and

$$
\begin{aligned}
& \sup _{\varepsilon \leq t \leq 1} \mathbb{E} \int_{|y| \geq N} e^{2(1-\nu) \gamma \varphi(y)}|\nabla u(t, y)|^{2} d y+\mathbb{E} \int_{\varepsilon}^{1} \int_{|y| \geq N} e^{2(1-\nu) \gamma \varphi(y)}\left|\mathcal{D}^{2} u(t, y)\right|^{2} d y d t \\
\leq & \frac{C}{\varepsilon} \mathbb{E} \int_{\varepsilon / 4}^{1} \int_{\mathbb{R}^{n}} e^{2 \gamma \varphi(y)} u^{2}(t, y) d y d t<\infty
\end{aligned}
$$

by the energy estimate in Lemma 2.1 .

Finally, we fix $N$ and $\nu$ such that $(2.9)$ and 2.10 hold, and without loss of generality, we may assume $N \geq \max \{\gamma, 2\}$. In this case, we have

$\mathbb{E} \int_{|x| \geq N} e^{2 \gamma \varphi_{a}(x)}|\nabla u(t)|^{2} d x=\mathbb{E} \int_{\{|x| \geq N\} \cap E_{a}} e^{2 \gamma \varphi_{a}(x)}|\nabla u(t)|^{2} d x+\mathbb{E} \int_{\{|x| \geq N\} \cap E_{a}^{c}} e^{2 \gamma \varphi_{a}(x)}|\nabla u(t)|^{2} d x$,

where $E_{a}=\left\{x \in \mathbb{R}^{n}: 2|x|^{2-a} \leq a+(2-a)(1-\nu)|x|^{2} /(1+4 \gamma)\right\}$, and $E_{a}^{c}$ is the complement of $E_{a}$.

For the first integral, we have that $\varphi_{a}(x) \leq(1-\nu) \varphi(x)$, where $\varphi$ is defined in (2.7), and thus,

$$
\mathbb{E} \int_{\{|x| \geq N\} \cap E_{a}} e^{2 \gamma \varphi_{a}(x)}|\nabla u(t)|^{2} d x \leq \mathbb{E} \int_{|x| \geq N} e^{2(1-\nu) \gamma \varphi(x)}|\nabla u(t, x)|^{2} d x
$$

which by 2.10 implies that

$$
\sup _{\varepsilon \leq t \leq 1} \mathbb{E} \int_{\{|x| \geq N\} \cap E_{a}} e^{2 \gamma \varphi_{a}(x)}|\nabla u(t)|^{2} d x<\infty
$$


For the second integral, we use the fact that if $x \in E_{a}^{c}$, then $|x|<2^{1 / a}[(1+4 \gamma) /(1-\nu)]^{1 / a}$ when $a \in(0,1)$. In fact,

$$
\begin{aligned}
& 2|x|^{2-a}>a+\frac{(2-a)(1-\nu)}{1+4 \gamma}|x|^{2} \\
\Leftrightarrow & \frac{(2-a)(1-\nu)}{1+4 \gamma}|x|^{a}<2-\frac{a}{|x|^{2-a}}<2 \\
\Rightarrow & |x|^{a}<\frac{2(1+4 \gamma)}{(2-a)(1-\nu)}<2 \frac{1+4 \gamma}{1-\nu} .
\end{aligned}
$$

Hence,

$$
\begin{aligned}
\mathbb{E} \int_{\{|x| \geq N\} \cap E_{a}^{c}} e^{2 \gamma \varphi_{a}(x)}|\nabla u(t, x)|^{2} d x & \leq \mathbb{E} \int_{\left.\{|x| \geq N\} \cap\left\{|x| \leq 2^{1 / a}[(1+4 \gamma) /(1-\nu)]\right]^{1 / a}\right\}} e^{2 \gamma \varphi_{a}(x)}|\nabla u(t)|^{2} d x \\
& \leq e^{2 \gamma \varphi_{a}\left(2^{1 / a}[(1+4 \gamma) /(1-\nu)]^{1 / a}\right)} \mathbb{E} \int_{|x| \geq N}|\nabla u(t, x)|^{2} d x,
\end{aligned}
$$

which by 2.9 implies that

$$
\sup _{\varepsilon \leq t \leq 1} \mathbb{E} \int_{\{|x| \geq N\} \cap E_{a}^{c}} e^{2 \gamma \varphi_{a}(x)}|\nabla u(t, x)|^{2} d x<\infty .
$$

It follows from 2.11, 2.12 and 2.13 that

$$
\sup _{\varepsilon \leq t \leq 1} \mathbb{E} \int_{|x| \geq N} e^{2 \gamma \varphi_{a}(x)}|\nabla u(t, x)|^{2} d x<\infty
$$

Similarly,

$$
\mathbb{E} \int_{\varepsilon}^{1} \int_{|x| \geq N} e^{2 \gamma \varphi_{a}(x)}\left|\mathcal{D}^{2} u(t, x)\right|^{2} d x<\infty
$$

For the space integral over the region $\{|x|<N\}$, all the estimates are obvious, and we finish the proof.

Remark 2.3. It is worthy to note that the estimates in Lemma 2.2 may blow up as a or $\varepsilon$ goes to zero, which indicates that we cannot use the energy estimate directly to work with $\sup _{t \in[0,1]} \mathbb{E}\left\|e^{\gamma|x|^{2}} u(t)\right\|$. However, we are going to use this lemma qualitatively but not quantitatively, so this fact is not an issue for our purpose.

\section{GAussian InTERIOR DECAY}

We first introduce a formal calculation to be used frequently in the sequel.

Lemma 3.1. Let $\mathcal{S}$ and $\mathcal{A}$ be a symmetric and a skew-symmetric operators, respectively, possibly dependent on the time variable. Suppose $V$ and $G$ are bounded stochastic processes in $[0,1] \times \mathbb{R}^{n}$, and a reasonable function $f(t, x)$ satisfies

$$
d f=(\mathcal{S}+\mathcal{A}) f d t+V f d t+G f d W(t) .
$$


TITLE WILL BE SET BY THE PUBLISHER

We also assume that there exists a time dependent operator $\mathcal{S}_{t}$ such that

$$
d(\mathcal{S} f)=\mathcal{S}_{t} f d t+\mathcal{S} d f
$$

Then there is a function $Q(t)$ and a universal constant $N$ such that

$$
\begin{gathered}
\frac{d^{2}}{d t^{2}}[\log H(t)+Q(t)] \geq \frac{2}{H(t)}\left\{\mathbb{E}\left(\mathcal{S}_{t} f+[\mathcal{S}, \mathcal{A}] f, f\right)+D_{G}(t)-\frac{D(t) H_{G}(t)}{H(t)}\right\}, \\
\|Q(t)\|_{\infty} \leq N\left(\|V\|_{\infty}+\|V\|_{\infty}^{2}+\|G\|_{\infty}^{2}\right),
\end{gathered}
$$

where

$$
\begin{aligned}
& H(t)=\mathbb{E}\|f\|^{2}, H_{G}(t)=\mathbb{E}\|G f\|^{2}, \\
& D(t)=\mathbb{E}(\mathcal{S} f, f), D_{G}(t)=\mathbb{E}(\mathcal{S}(G f), G f) .
\end{aligned}
$$

Proof. By Itô's formula, we have

$$
\begin{aligned}
d(f, f) & =2(d f, f)+\|G f\|^{2} d t \\
& =2(\mathcal{S} f, f) d t+2(V f, f) d t+2(G f, f) d W .
\end{aligned}
$$

Thus

$$
\dot{H}(t)=2 D(t)+2 \mathbb{E}(V f, f)+H_{G}(t)
$$

Let us rewrite $D$ as follows:

$$
D(t)=\frac{1}{2} \mathbb{E}(2 \mathcal{S} f+V f, f)-\frac{1}{2} \mathbb{E}(V f, f)
$$

and so

$$
\dot{H}(t)=\mathbb{E}(2 \mathcal{S} f+V f, f)+\mathbb{E}(V f, f)+H_{G}(t) .
$$

Then

$$
D(t) \dot{H}(t)=\frac{1}{2}\left[|\mathbb{E}(2 \mathcal{S} f+V f, f)|^{2}-|\mathbb{E}(V f, f)|^{2}\right]+D(t) H_{G}(t) .
$$

By Itô's formula again and equality (3.1), we have

$$
\begin{aligned}
d(\mathcal{S} f, f)= & (d(\mathcal{S} f), f)+(\mathcal{S} f, d f)+(\mathcal{S}(G f), G f) d t \\
= & \left(\mathcal{S}_{t} f, f\right) d t+2(\mathcal{S} f, d f)+(\mathcal{S}(G f), G f) d t \\
= & \left(\mathcal{S}_{t} f, f\right) d t+2\|\mathcal{S} f\|^{2} d t+2(\mathcal{S} f, \mathcal{A} f) d t+2(\mathcal{S} f, V f) d t \\
& +2(\mathcal{S} f, G f) d W+(\mathcal{S}(G f), G f) d t \\
= & \left(\mathcal{S}_{t} f, f\right) d t+([\mathcal{S}, \mathcal{A}] f, f) d t+\frac{1}{2}\|2 \mathcal{S} f+V f\|^{2} d t-\frac{1}{2}\|V f\|^{2} d t \\
& +2(\mathcal{S} f, G f) d W+(\mathcal{S}(G f), G f) d t
\end{aligned}
$$

In the last equality, we use the identities

$$
2(\mathcal{S} f, \mathcal{A} f)=([\mathcal{S}, \mathcal{A}] f, f),
$$

and

$$
2\|\mathcal{S} f\|^{2}+2(\mathcal{S} f, V f)=\frac{1}{2}\|2 \mathcal{S} f+V f\|^{2}-\frac{1}{2}\|V f\|^{2} .
$$


Thus

$$
\dot{D}(t)=\mathbb{E}\left(\mathcal{S}_{t} f+[\mathcal{S}, \mathcal{A}] f, f\right)+\frac{1}{2} \mathbb{E}\|2 \mathcal{S} f+V f\|^{2}-\frac{1}{2} \mathbb{E}\|V f\|^{2}+D_{G}(t) .
$$

Thus by 3.5 we obtain

$$
\frac{d}{d t}[\log H(t)]=\frac{\dot{H}(t)}{H(t)}=\frac{2 D(t)}{H(t)}-\dot{F}
$$

where $F$ verifies

$$
\dot{F}(t)=-\frac{2 \mathbb{E}(V f, f)}{H(t)}-\frac{H_{G}(t)}{H(t)}, \quad F(0)=0 .
$$

Then it follows from 3.8 and 3.9 that

$$
\begin{aligned}
& \frac{d^{2}}{d t^{2}}[\log H(t)+F(t)] \\
= & 2 \frac{d}{d t}\left[\frac{D(t)}{H(t)}\right]=\frac{2}{H(t)}\left[\dot{D}(t)-\frac{D(t) \dot{H}(t)}{H(t)}\right] \\
= & \frac{2}{H(t)}\left\{\mathbb{E}\left(\mathcal{S}_{t} f+[\mathcal{S}, \mathcal{A}] f, f\right)+\frac{1}{2} \mathbb{E}\|2 \mathcal{S} f+V f\|^{2}-\frac{1}{2} \mathbb{E}\|V f\|^{2}+D_{G}(t)\right. \\
& \left.\quad-\frac{1}{2 H(t)}\left[|\mathbb{E}(2 \mathcal{S} f+V f, f)|^{2}-|\mathbb{E}(V f, f)|^{2}\right]-\frac{D(t) H_{G}(t)}{H(t)}\right\} .
\end{aligned}
$$

By Cauchy-Schwarz inequality, we have

$$
|\mathbb{E}(2 \mathcal{S} f+V f, f)|^{2} \leq \mathbb{E}\|2 \mathcal{S} f+V f\|^{2} \mathbb{E}\|f\|^{2},
$$

which, together with the inequalities

$$
|\mathbb{E}(V f, f)|^{2} \geq 0, \quad \mathbb{E}\|V f\|^{2} \leq\|V\|_{\infty}^{2} \mathbb{E}\|f\|^{2},
$$

implies

$$
\frac{d^{2}}{d t^{2}}[\log H(t)+F(t)] \geq \frac{2}{H(t)}\left\{\mathbb{E}\left(\mathcal{S}_{t} f+[\mathcal{S}, \mathcal{A}] f, f\right)+D_{G}(t)-\frac{D(t) H_{G}(t)}{H(t)}\right\}-\|V\|_{\infty}^{2} .
$$

Now let $Q(t)=F(t)+t(1-t)\|V\|_{\infty}^{2}$, and then 3.10 yields 3.2 and 3.3 , which completes the proof.

Remark 3.2. If the noise $G$ is deterministic and independent of the space variable $x$, the term $D_{G}(t)-D(t) H_{G}(t) / H(t)$ on (3.2), will disappear as long as $\mathcal{S}$ does not involve derivatives with respect to time, as will be our case. Thus, we can deal with the commutator part as the deterministic case in [10], and get rid of Assumption [1.1 as well as the restriction on $\gamma$ to arrive at a logarithmic convexity result analogous to Lemma 3 in [10]. Moreover, it is easy to show that the noise does not play a role if one tries to prove uniqueness from the logarithmic convexity estimate, so for spaceindependent deterministic noise the deterministic result still holds in the stochastic setting. Notice that $G \equiv 0$ is trivially a space-independent noise, so the deterministic result is covered. 
Recall that according to Remark 2.3, we are unable to control the term $\sup _{t \in[0,1]} \mathbb{E}\left\|e^{\gamma|x|^{2}} u(t)\right\|^{2}$ directly from the energy estimate. On the other hand, we cannot use this abstract result in the same way as in the deterministic case to control that quantity. However, the next lemma allows us to bound the corresponding $L^{2}$-norm in the time variable. Moreover, it also shows that both

$$
\mathbb{E}\left\|e^{\gamma|x|^{2}}|x| u(t)\right\|^{2}, \quad \text { and } \quad \mathbb{E}\left\|e^{\gamma|x|^{2}} \nabla u(t)\right\|^{2}
$$

are finite for almost every $t \in[0,1]$.

In the rest of this section, we will make another assumption on the potential $V$ and the noise $G$, due to the stochastic conformal transformation studied in Lemma 4.1.

Assumption 3.3. The measurable functions $V$ and $G: \Omega \times[0,1] \times \mathbb{R}^{n} \rightarrow \mathbb{R}$ satisfy the following conditions:

(1) $V, G$ and $\nabla G$ are bounded on $\Omega \times[0,1] \times \mathbb{R}^{n}$;

(2) Given $\gamma>0$, there exists $\varepsilon>0$ such that $\sup |G(\omega, t, x)| \leq \sqrt{\gamma}|x|^{-\varepsilon}$ for $|x| \geq \max \{\gamma, 2\}$;

(3) $\lim _{L \rightarrow \infty} \sup _{\substack{t \in[0,1],|x|>L \\ \omega \in \Omega}}|V(\omega, t, x)|=\lim _{L \rightarrow \infty} \sup _{\substack{t \in[0,1],|x|>L \\ \omega \in \Omega}}^{\substack{t \in[0,1] \\ \omega \in \Omega}}|\nabla G(\omega, t, x)|=0$.

Lemma 3.4. Suppose $u$ is a solution of equation (1.1), and Assumption 3.3 holds. Then, for $\gamma>\|G\|_{\infty}^{2} / 4$, there is $N=N\left(\gamma,\|V\|_{\infty},\|G\|_{\infty},\|\nabla G\|_{\infty}\right)$ such that

$$
\begin{array}{r}
\int_{0}^{1} \mathbb{E}\left\|e^{\gamma|x|^{2}} u(t)\right\|^{2} d t+\int_{0}^{1} t(1-t) \mathbb{E}\left\|e^{\gamma|x|^{2}}|x| u(t)\right\|^{2} d t+\int_{0}^{1} t(1-t) \mathbb{E}\left\|e^{\gamma|x|^{2}} \nabla u(t)\right\|^{2} d t \\
\leq N\left(\mathbb{E}\left\|e^{\gamma|x|^{2}} u(0)\right\|^{2}+\mathbb{E}\left\|e^{\gamma|x|^{2}} u(1)\right\|^{2}+\sup _{t \in[0,1]} \mathbb{E}\|u(t)\|^{2}\right)
\end{array}
$$

Proof. Let $f=e^{\gamma \varphi} u$, where $\varphi=\varphi(x)$ is to be chosen. Then $f$ satisfies, formally

$$
d f=(\mathcal{S} f+\mathcal{A} f+V f) d t+G f d W(t),
$$

where

$$
\mathcal{S}=\Delta+\gamma^{2}|\nabla \varphi|^{2}, \quad \text { and } \quad \mathcal{A}=-2 \gamma \nabla \varphi \cdot \nabla-\gamma \Delta \varphi
$$

are symmetric and skew-symmetric operators, respectively. We do calculations as in Lemma 3.1 . and recall that

$$
\begin{aligned}
& \dot{H}(t)=2 D(t)+2 \mathbb{E}(V f, f)+H_{G}(t), \quad \text { and } \\
& \dot{D}(t)=\mathbb{E}([\mathcal{S}, \mathcal{A}] f, f)+\frac{1}{2} \mathbb{E}\|2 \mathcal{S} f+V f\|^{2}-\frac{1}{2} \mathbb{E}\|V f\|^{2}+D_{G}(t),
\end{aligned}
$$

where $H, H_{G}, D$ and $D_{G}$ are defined in 3.4 .

Multiplying $\dot{H}(t)$ by $(1-2 t)$ and integrating in $t \in[0,1]$, we get

$$
\int_{0}^{1}(1-2 t) \dot{H}(t) d t=2 \int_{0}^{1} H(t) d t-H(0)-H(1) .
$$


On the other hand,

$$
\begin{aligned}
& \int_{0}^{1}(1-2 t) \dot{H}(t) d t \\
= & 2 \int_{0}^{1}(1-2 t) D(t) d t+2 \int_{0}^{1}(1-2 t) \mathbb{E}(V f, f) d t+\int_{0}^{1}(1-2 t) H_{G}(t) d t \\
= & 2 \int_{0}^{1} D(t) d(t(1-t))+2 \int_{0}^{1}(1-2 t) \mathbb{E}(V f, f) d t+\int_{0}^{1}(1-2 t) H_{G}(t) d t \\
= & -2 \int_{0}^{1} t(1-t) \dot{D}(t) d t+2 \int_{0}^{1}(1-2 t) \mathbb{E}(V f, f) d t+\int_{0}^{1}(1-2 t) H_{G}(t) d t \\
= & 2 \int_{0}^{1} t(1-t) \mathbb{E}([\mathcal{S}, \mathcal{A}] f, f) d t-\int_{0}^{1} t(1-t) \mathbb{E}\|2 \mathcal{S} f+V f\|^{2} d t \\
& \quad+\int_{0}^{1} t(1-t) \mathbb{E}\|V f\|^{2} d t-2 \int_{0}^{1} t(1-t) D_{G}(t) d t \\
& +2 \int_{0}^{1}(1-2 t) \mathbb{E}(V f, f) d t+\int_{0}^{1}(1-2 t) H_{G}(t) d t .
\end{aligned}
$$

Therefore, we have

$$
\begin{aligned}
H(0)+ & H(1)-2 \int_{0}^{1} H(t) d t \\
& \geq 2 \int_{0}^{1} t(1-t) \mathbb{E}([\mathcal{S}, \mathcal{A}] f, f) d t-\int_{0}^{1} t(1-t) \mathbb{E}\|V f\|^{2} d t-2 \int_{0}^{1}(1-2 t) \mathbb{E}(V f, f) d t \\
& -\int_{0}^{1}(1-2 t) H_{G}(t) d t+2 \int_{0}^{1} t(1-t) D_{G}(t) d t .
\end{aligned}
$$

Now, formally, if $\varphi(x)=|x|^{2}$ we have from 3.11 that

$$
([\mathcal{S}, \mathcal{A}] f, f)=8 \gamma \int_{\mathbb{R}^{n}}|\nabla f|^{2} d x+32 \gamma^{3} \int_{\mathbb{R}^{n}}|x|^{2}|f|^{2} d x
$$

and

$$
\begin{aligned}
(\mathcal{S}(G f), G f) & =-\int_{\mathbb{R}^{n}}|\nabla(G f)|^{2} d x+4 \gamma^{2} \int_{R^{n}}|x|^{2}|G f|^{2} d x \\
& \geq-2 \int_{\mathbb{R}^{n}}|G|^{2}|\nabla f|^{2} d x-2 \int_{\mathbb{R}^{n}}|\nabla G|^{2}|f| d x .
\end{aligned}
$$


Thus,

$$
\begin{array}{rl}
2 \int_{0}^{1} & H(t) d t+4\left(4 \gamma-\|G\|_{\infty}^{2}\right) \int_{0}^{1} t(1-t) \mathbb{E} \int_{\mathbb{R}^{n}}|\nabla f|^{2} d x d t \\
& +64 \gamma^{3} \int_{0}^{1} t(1-t) \mathbb{E} \int_{\mathbb{R}^{n}}|x|^{2}|f|^{2} d x d t \\
\leq H(0) & +H(1)+\int_{0}^{1} t(1-t) \mathbb{E}\|V f\|^{2} d t+2 \int_{0}^{1}(1-2 t) \mathbb{E}(V f, f) d t \\
& +\int_{0}^{1}(1-2 t) H_{G}(t) d t+4 \int_{0}^{1} t(1-t) \mathbb{E} \int_{\mathbb{R}^{n}}|\nabla G|^{2}|f|^{2} d x d t .
\end{array}
$$

By assumptions on $G$ and $V$, for a given $\varepsilon>0$, there exists $L>0$ such that when $|x|>L$ we have

$$
\max \left\{\sup _{\substack{t \in[0,1] \\ \omega \in \Omega}}|G(\omega, t, x)|, \sup _{\substack{t \in[0,1] \\ \omega \in \Omega}}|V(\omega, t, x)| \sup _{\substack{t \in[0,1] \\ \omega \in \Omega}}|\nabla G(\omega, t, x)|\right\} \leq \varepsilon .
$$

Therefore, we obtain that

$$
\begin{aligned}
\int_{0}^{1} t(1-t) \mathbb{E}\|V f\|^{2} d t & =\int_{0}^{1} t(1-t) \mathbb{E}\left[\int_{|x| \leq L}|V|^{2}|f|^{2} d x+\int_{|x|>L}|V|^{2}|f|^{2} d x\right] d t \\
& \leq \frac{\|V\|_{\infty}^{2}}{4} e^{2 \gamma L^{2}} \sup _{t \in[0,1]} \mathbb{E}\|u(t)\|^{2}+\frac{\varepsilon^{2}}{4} \int_{0}^{1} H(t) d t
\end{aligned}
$$

In the same way, we also have

$$
\begin{gathered}
2 \int_{0}^{1}(1-2 t) \mathbb{E}(V f, f) d t \leq 2\|V\|_{\infty} e^{2 \gamma L^{2}} \sup _{t \in[0,1]} \mathbb{E}\|u(t)\|^{2}+2 \varepsilon \int_{0}^{1} H(t) d t, \\
\int_{0}^{1}(1-2 t) H_{G}(t) d t \leq\|G\|_{\infty}^{2} e^{2 \gamma L^{2}} \sup _{t \in[0,1]} \mathbb{E}\|u(t)\|^{2}+\varepsilon^{2} \int_{0}^{1} H(t) d t, \\
4 \int_{0}^{1} t(1-t) \mathbb{E} \int_{\mathbb{R}^{n}}|\nabla G|^{2}|f|^{2} d x d t \leq\|\nabla G\|_{\infty}^{2} e^{2 \gamma L^{2}} \sup _{t \in[0,1]} \mathbb{E}\|u(t)\|^{2}+\varepsilon^{2} \int_{0}^{1} H(t) d t .
\end{gathered}
$$

Putting everything together yields

$$
\begin{aligned}
& \quad\left(2-9 \varepsilon^{2} / 4-2 \varepsilon\right) \int_{0}^{1} H(t) d t+4\left(4 \gamma-\|G\|_{\infty}^{2}\right) \int_{0}^{1} t(1-t) \mathbb{E} \int_{\mathbb{R}^{n}}|\nabla f|^{2} d x d t \\
& \quad+64 \gamma^{3} \int_{0}^{1} t(1-t) \mathbb{E} \int_{\mathbb{R}^{n}}|x|^{2}|f|^{2} d x d t \\
& \leq H(0)+H(1)+N \sup _{t \in[0,1]} \mathbb{E}\|u(t)\|^{2} .
\end{aligned}
$$


We can now choose $\varepsilon$ small enough so that $2-9 \varepsilon^{2} / 4-2 \varepsilon>0$ and conclude the result by using the inequality $(2.21)$ in 10

$$
2 \mathbb{E} \int_{\mathbb{R}^{n}}|\nabla f|^{2}+4 \gamma^{2}|x|^{2}|f|^{2} d x \geq \mathbb{E} \int_{\mathbb{R}^{n}} e^{2 \gamma|x|^{2}}|\nabla u|^{2} d x .
$$

In order to make the calculations above rigorous, we set $f_{a}=e^{\gamma \varphi_{a}} u$, where $\varphi_{a}$ satisfies 2.2 and (2.3). Then

and thus

$$
\partial_{i j} \varphi_{a}(x)=\frac{\varphi_{a}^{\prime}(|x|)}{|x|} \delta_{i j}-a \frac{x_{i} x_{j}}{|x|^{2}} \zeta_{a}(|x|),
$$

$$
\mathcal{D}^{2} \varphi_{a}(x)= \begin{cases}2 I_{n}+O(a) \sum_{i, j=1}^{n} E_{i j}, & \text { if } 0 \leq|x| \leq \max \{\gamma, 2\}, \\ 2|x|^{-a} I_{n}+O(a) \sum_{i, j=1}^{n} E_{i j}, & \text { if }|x| \geq \max \{\gamma, 2\}\end{cases}
$$

where $I_{n}$ is an $n \times n$ identity matrix, and $E_{i j}$ is the elementary matrix whose only nonzero entry is a 1 in $i$-th row and $j$-th column. Also, in this case we have

$$
\left\|\Delta^{2} \varphi_{a}\right\|_{\infty} \leq C(n) a .
$$

Thus,

$$
\begin{aligned}
\int_{\mathbb{R}^{n}} \mathcal{D}^{2} \varphi_{a} \nabla f_{a} \cdot \nabla f_{a} d x= & \int_{|x| \leq \max \{\gamma, 2\}} \mathcal{D}^{2} \varphi_{a} \nabla f_{a} \cdot \nabla f_{a} d x+\int_{|x| \geq \max \{\gamma, 2\}} \mathcal{D}^{2} \varphi_{a} \nabla f_{a} \cdot \nabla f_{a} d x \\
\geq & 2 \int_{|x| \leq \max \{\gamma, 2\}}\left|\nabla f_{a}\right|^{2} d x+n \int_{|x| \leq \max \{\gamma, 2\}} O(a)\left|\nabla f_{a}\right|^{2} d x \\
& +2 \int_{|x| \geq \max \{\gamma, 2\}}|x|^{-a}\left|\nabla f_{a}\right|^{2} d x+n \int_{|x| \geq \max \{\gamma, 2\}} O(a)\left|\nabla f_{a}\right|^{2} d x, \quad(3.1)
\end{aligned}
$$

and so

$$
\begin{aligned}
& \quad 8 \gamma \int_{\mathbb{R}^{n}} \mathcal{D}^{2} \varphi_{a} \nabla f_{a} \cdot \nabla f_{a} d x-4 \int_{\mathbb{R}^{n}}|G|^{2}\left|\nabla f_{a}\right|^{2} d x \\
& \geq 4 \int_{|x| \leq \max \{\gamma, 2\}}\left(4 \gamma+n O(a)-\|G\|_{\infty}^{2}\right)\left|\nabla f_{a}\right|^{2} d x \\
& \quad+4 \int_{|x| \geq \max \{\gamma, 2\}}(3 \gamma+n O(a))|x|^{-a}\left|\nabla f_{a}\right|^{2} d x+4 \int_{|x| \geq \max \{\gamma, 2\}}\left(\gamma|x|^{-a}-|G|^{2}\right)\left|\nabla f_{a}\right|^{2} d x .
\end{aligned}
$$

By choosing $a$ small enough, the first two integrals on the right hand side of the above inequality are non-negative by the condition that $\gamma>\|G\|_{\infty}^{2} / 4$, and so is the last one due to the decay of the noise $G$ in Assumption 3.3 .

Observing that

$$
\begin{aligned}
\left([\mathcal{S}, \mathcal{A}] f_{a}, f_{a}\right)=4 \gamma \int_{\mathbb{R}^{n}} \mathcal{D}^{2} \varphi_{a} \nabla f_{a} \cdot \nabla f_{a} d x+4 \gamma^{3} \int_{\mathbb{R}^{n}} \mathcal{D}^{2} \varphi_{a} \nabla \varphi_{a} \cdot \nabla \varphi_{a}\left|f_{a}\right|^{2} d x & \\
& -\gamma \int_{\mathbb{R}^{n}} \Delta^{2} \varphi_{a}\left|f_{a}\right|^{2} d x
\end{aligned}
$$


and repeating the formal computations as before, we obtain

$$
\begin{gathered}
\left(2-9 \varepsilon^{2} / 4-2 \varepsilon-\gamma C(n) a\right) \int_{0}^{1} H_{a}(t) d t+4 \gamma^{3} \int_{0}^{1} t(1-t) \mathbb{E} \int_{\mathbb{R}^{n}} \mathcal{D}^{2} \varphi_{a} \nabla \varphi_{a} \cdot \nabla \varphi_{a}\left|f_{a}\right|^{2} d x d t \\
+4 \int_{|x| \leq \max \{\gamma, 2\}}\left(4 \gamma+n O(a)-\|G\|_{\infty}^{2}\right)\left|\nabla f_{a}\right|^{2} d x+4 \int_{|x| \geq \max \{\gamma, 2\}}(3 \gamma+n O(a))|x|^{-a}\left|\nabla f_{a}\right|^{2} d x \\
+4 \int_{|x| \geq \max \{\gamma, 2\}}\left(\gamma|x|^{-a}-|G|^{2}\right)\left|\nabla f_{a}\right|^{2} d x \\
\leq H_{a}(0)+H_{a}(1)+N \sup _{t \in[0,1]} \mathbb{E}\|u(t)\|^{2},
\end{gathered}
$$

where $H_{a}(t)=\mathbb{E}\left\|f_{a}(t)\right\|^{2}$. By letting $a$ tend to zero, we prove $(3.13$ rigorously.

Next, we would like to replace the term $\int_{\mathbb{R}^{n}}|\nabla f|^{2} d x$ by $\int_{\mathbb{R}^{n}} e^{2 \gamma|x|^{2}}|\nabla u|^{2} d x$ in $(3.13)$. To do this, we notice that $\left(3.13\right.$ holds for $f_{\rho}=e^{(\gamma-\rho)|x|^{2}} u$ as well. Then by using the same argument as the interior regularity result in Lemma 2.2 , we can justify $(3.14)$ with such $f_{\rho}$ for $t \in[\varepsilon, 1]$. In the end, we send $\rho$ and $\varepsilon$ to zero and complete the proof.

Remark 3.5. Thanks to the decay of $V$ and $G$ in Assumption 1.1 for any $\epsilon>0$ there exists $L>0$ such that (3.12) holds when $|x|>L$. However, we use this fact for $\epsilon>0$ so that $2-9 \epsilon^{2} / 4-2 \epsilon>0$. This implies that condition (3) in Assumption 1.1 could be replaced by

$$
\exists L>0 \text { such that } \sup _{\substack{t \in[0,1],|x|>L \\ \omega \in \Omega}}|V(\omega, x, t)|, \sup _{\substack{t \in[0,1],|x|>L \\ \omega \in \Omega}}|\nabla G(\omega, x, t)|<c
$$

for some universal $c>0$. Hence, no decay is really needed in $V$ and $\nabla G$ and what we really need is a small enough bound for $V$ and $\nabla G$ far from the origin. Notice that the decay condition (2) in Assumption 1.1 for $G$ is also needed in our justification argument, so we are not allowed to remove this decay.

Remark 3.6. In order to show that the commutator is positive we have to absorb the integral $\int|G|^{2}\left|\nabla f_{a}\right|^{2} d x$ in one of the positive terms appearing in the commutator. According to (3.18) our only chance is to use the positive integral $\int|x|^{-a}\left|\nabla f_{a}\right|^{2} d x$ and hence we assume that the noise $G$ has a polynomial decay (condition (2) in Assumption 1.1).

\section{Proof of main Result}

It is noted that in Lemma 3.4 we require that the solution $u$ has the same quadratic exponential decay for $t_{0}=0$ and $t_{1}=1$, but Theorem 1.3 assumes no decay for the initial data. In order to overcome this issue, we introduce the following conformal transformation, also known as Appell transformation for our stochastic equation.

Lemma 4.1. Assume $u(t, x)$ verifies

$$
d u=(\Delta u+V(t, x) u) d t+G(t, x) u d W(t), \quad(t, x) \in[0,1] \times \mathbb{R}^{n} .
$$

Let $\alpha, \beta>0$ and set

$$
y(t, x)=[a(t)]^{\frac{n}{2}} u(b(t), a(t) x) e^{\frac{a(t) \kappa|x|^{2}}{4}},
$$


where

then $y$ verifies

$$
\kappa=\sqrt{\frac{\alpha}{\beta}}-\sqrt{\frac{\beta}{\alpha}}, \quad a(t)=\frac{\sqrt{\alpha \beta}}{\alpha(1-t)+\beta t} \quad \text { and } \quad b(t)=\sqrt{\frac{\beta}{\alpha}} a(t) t .
$$

with

$$
d y=(\Delta y+\widetilde{V} y) d t+\widetilde{g} y d W(b(t))
$$

Moreover, for any $\gamma \in \mathbb{R}$,

$$
\widetilde{V}(t, x)=[a(t)]^{2} V(b(t), a(t) x), \quad \text { and } \quad \widetilde{g}(t, x)=G(b(t), a(t) x) .
$$

$$
\mathbb{E}\left\|e^{\gamma|\cdot|^{2}} y(t)\right\|^{2}=\mathbb{E}\left\|e^{\left(\gamma a^{2}(1-s)+\kappa a(1-s) / 4\right)|\cdot|^{2}} u(s)\right\|^{2},
$$

where $s=b(t)$.

Proof. By Itô's formula,

$$
d y=\frac{n}{2} a^{\frac{n}{2}-1} a^{\prime} u e^{\frac{a \kappa|x|^{2}}{4}} d t+a^{\frac{n}{2}} e^{\frac{a \kappa|x|^{2}}{4}} d u(b(t), a(t) x)+a^{\frac{n}{2}} a^{\prime} \frac{\kappa|x|^{2}}{4} e^{\frac{a \kappa|x|^{2}}{4}} u d t,
$$

where

$$
d u(b(t), a(t) x)=\left.d u(b(t), z)\right|_{z=a(t) x}+\left.d u(\tau, a(t), x)\right|_{\tau=b(t)} .
$$

On one hand, observing that

$$
\begin{aligned}
u(b(t), z) & =u(0, z)+\int_{0}^{b(t)}[\Delta u+V u](s, z) d s+\int_{0}^{b(t)} G u(s, z) d W(s) \\
& =u(0, z)+\int_{0}^{t}[\Delta u+V u](b(s), z) b^{\prime}(s) d s+\int_{0}^{t} G u(b(s), z) d W(b(s)),
\end{aligned}
$$

where the second equality follows from the time change formula for Brownian motions, or more generally, local martingales, see for example [23. Proposition 1.5, page 181], and

$$
d u(\tau, a(t) x)=a^{\prime}(t) \nabla u(\tau, a(t) x) \cdot x d t,
$$

we have

$$
a^{-\frac{n}{2}} e^{-\frac{a \kappa|x|^{2}}{4}} d y=\frac{n}{2} a^{-1} a^{\prime} u d t+\left[(\Delta u+V u) b^{\prime}+a^{\prime} \nabla u \cdot x+a^{\prime} \frac{\kappa|x|^{2}}{4} u\right] d t+G u d W(b(t)) .
$$

On the other hand,

$$
a^{-\frac{n}{2}} e^{-\frac{a \kappa|x|^{2}}{4}} \Delta y=a^{2} \Delta u+\kappa a^{2} \nabla u \cdot x+\frac{n \kappa}{2} a u+\frac{\kappa^{2}}{4} a^{2}|x|^{2} u .
$$

Then it follows from 4.4 and 4.5 that

$$
\begin{aligned}
& a^{-\frac{n}{2}} e^{-\frac{a \kappa|x|^{2}}{4}}(d y-\Delta y d t) \\
&=\left[\left(b^{\prime}-a^{2}\right) \Delta u+\left(a^{\prime}-\kappa a^{2}\right) \nabla \cdot x+\frac{n}{2} a^{-1}\left(a^{\prime}-\kappa a^{2}\right) u+\frac{\kappa}{4}\left(a^{\prime}-a\right)|x|^{2} u\right] d t \\
&+b^{\prime} V u d t+G u d W(b(t)) .
\end{aligned}
$$


Using the identities $a^{\prime}=\kappa a^{2}$ and $b^{\prime}=a^{2}$, we obtain (4.2). Then relation 4.3 is a consequence of the transform (4.1) and the fact that $a(t) a(s)=1$.

Remark 4.2. In Lemma 4.1, if $u$ is $\mathcal{F}_{t}$-adapted, then y defined in 4.2 is $\mathcal{F}_{b(t)}$-adapted.

It is known that the conformal transformation $y$ is equivalent in probability law to the process $\widetilde{u}$ satisfying

where

$$
d \widetilde{u}=(\Delta \widetilde{u}+\widetilde{V}(t, x) \widetilde{u}) d t+\widetilde{G}(t, x) \widetilde{u} d \widetilde{W}(t)
$$

$$
\widetilde{V}(t, x)=[a(t)]^{2} V(b(t), a(t) x), \quad \widetilde{G}(t, x)=G(b(t), a(t) x) \sqrt{b^{\prime}(t)},
$$

and $\widetilde{W}$ is another Wiener process. Since the norms we consider are under the probability expectation, we may by a slight abuse of notation denote by $\widetilde{u}$ the conformal transformation of $u$. Then we have

$$
\mathbb{E}\left\|e^{\gamma|x|^{2}} \widetilde{u}(0)\right\|^{2}=\mathbb{E}\|u(0)\|^{2}, \quad \mathbb{E}\left\|e^{\gamma|x|^{2}} \widetilde{u}(1)\right\|^{2}=\mathbb{E}\left\|e^{|x|^{2} / \delta^{2}} u(1)\right\|^{2},
$$

by choosing $\alpha=1, \beta=1+4 \gamma$ and $\gamma=1 /(2 \delta)$ in Lemma 4.1 . We also have

$$
\|\widetilde{V}\|_{\infty} \leq(1+4 \gamma)\|V\|_{\infty}, \quad\|\widetilde{G}\|_{\infty} \leq \sqrt{1+4 \gamma}\|G\|_{\infty}, \quad \text { and } \quad\|\nabla \widetilde{G}\|_{\infty} \leq(1+4 \gamma)\|\nabla G\|_{\infty}
$$

by the identities $b^{\prime}=a^{2}$ and $\|a\|_{\infty}=\sqrt{1+4 \gamma}$.

Finally, we are ready to prove our main theorem.

Proof of Theorem 1.3. We consider $\gamma=1 /(2 \delta)>1 / 2$ and apply to the solution $u$ the conformal transformation in Lemma 4.1. It follows from (4.7) that $\widetilde{V}$ and $\widetilde{G}$ satisfy Assumption 3.3 as long as $V$ and $G$ satisfy Assumption 1.1 and that $\|\widetilde{G}\|_{\infty}^{2}<4 \gamma$. Therefore, we can apply previous lemmas and the interior regularity to show that the subsequent formal computations are correct.

For $R>0$ and $M \geq R$ we define the functions

$$
\theta_{M}(x)=\left\{\begin{array}{ll}
0, & |x|>2 M, \\
1, & |x| \leq M,
\end{array} \quad \eta_{R}(x)= \begin{cases}1, & t \in\left[\frac{1}{R}, 1-\frac{1}{R}\right], \\
0, & t \in\left[0, \frac{1}{2 R}\right] \cup\left[1-\frac{1}{2 R}, 1\right]\end{cases}\right.
$$

and consider $v(x, t)=\theta_{M}(x) \eta_{R}(t) \widetilde{u}(x, t)$. For $\gamma>\frac{1}{2}$ we define $\mu$ and $\epsilon>0$ such that

$$
\frac{\sqrt{1+\epsilon}}{2(1-\epsilon)}<\mu \leq \frac{\gamma}{1+\epsilon}
$$

and $f=e^{\varphi} v$ where

$$
\varphi(x, t)=\mu\left|x+R t(1-t) e_{1}\right|^{2}+\frac{R^{2} t(1-t)(12 t)}{6}-\frac{(1+\epsilon) R^{2} t(1-t)}{16 \mu} .
$$

We observe that

$$
\begin{aligned}
& d v=\theta_{M} \eta_{R}^{\prime \prime} \widetilde{u} d t+\theta_{M} \eta_{R} \Delta \widetilde{u} d t+\widetilde{V} v d t+\widetilde{G} v d \widetilde{W} \\
& \Delta v=\Delta \theta_{M} \eta_{R} \widetilde{u}+\theta_{M} \eta_{R} \Delta \widetilde{u}+2 \nabla \theta_{M} \eta_{R} \cdot \nabla \widetilde{u}
\end{aligned}
$$

Hence,

$$
d v-\Delta v d t=F d t+\widetilde{V} v d t+\widetilde{G} v d \widetilde{W},
$$

where $F:=\theta_{M} \eta_{R}^{\prime} \widetilde{u}-\left(2 \nabla \theta_{M} \nabla \widetilde{u}+\Delta \theta_{M} \widetilde{u}\right) \eta_{R}$. 
Now we write

$$
e^{\varphi}(d v-\Delta v d t)=d f-(\mathcal{S}+\mathcal{A}) f d t=e^{\varphi} F d t+\widetilde{V} f d t+\widetilde{G} f d \widetilde{W}
$$

where $\mathcal{S}$ and $\mathcal{A}$ are symmetric and skew-symmetric operators respectively. A computation shows (see [7, Lemma 8])

$$
\begin{aligned}
\mathcal{S}= & \Delta+4 \mu^{2}\left|x+R t(1-t) e_{1}\right|^{2}+2 \mu R(1-2 t)\left(x_{1}+R t(1-t)\right. \\
& +\left(t^{2}-t+\frac{1}{6}\right) R^{2}-\frac{(1+\epsilon) R^{2}(1-2 t)}{16 \mu}, \\
\mathcal{A}= & -4 \mu\left(x+R t(1-t) e_{1}\right) \cdot \nabla-2 \mu n, \\
\left(\mathcal{S}_{t} f+[\mathcal{S}, \mathcal{A}] f, f\right)= & 32 \mu^{3} \int_{\mathbb{R}^{n}}\left|x+R t(1-t) e_{1}+\frac{(4 \mu(1-2 t)-1) R}{16 \mu^{2}} e_{1}\right|^{2}|f|^{2} d x \\
+ & 8 \mu \int_{\mathbb{R}^{n}}|\nabla f|^{2} d x+\frac{\epsilon R^{2}}{8 \mu} \int_{\mathbb{R}^{n}}|f|^{2} d x .
\end{aligned}
$$

Let us multiply 4.8 by $-2 \mathcal{S} f$, then integrate in space and time and take expectation to get

$$
-2 \mathbb{E} \int_{0}^{1}(d f, \mathcal{S} f) d t+2 \mathbb{E} \int_{0}^{1}\|\mathcal{S} f\|^{2} d t+2 \mathbb{E} \int_{0}^{1}(\mathcal{A} f, \mathcal{S} f) d t=-2 \mathbb{E} \int_{0}^{1}\left(e^{\varphi} F+\widetilde{V} f, \mathcal{S} f\right) d t .
$$

Using that $d(\mathcal{S} f, f)=\left(\mathcal{S}_{t} f, f\right) d t+2(\mathcal{S} f, d f)+(S(\widetilde{G} f), \widetilde{G} f) d t$ the latter is equivalent to (remember that $f$ is of compact support)

$$
2 \mathbb{E} \int_{0}^{1}\|\mathcal{S} f\|^{2} d t+\mathbb{E} \int_{0}^{1}\left(\mathcal{S}_{t} f+[\mathcal{S}, \mathcal{A}] f, f\right) d t+\mathbb{E} \int_{0}^{1}(\mathcal{S}(\widetilde{G} f), \widetilde{G} f) d t=-2 \mathbb{E} \int_{0}^{1}\left(e^{\varphi} F+\widetilde{V} f, \mathcal{S} f\right) d t
$$

and, using Cauchy-Schwartz we arrive to

$$
\mathbb{E} \int_{0}^{1}\|\mathcal{S} f\|^{2} d t+\mathbb{E} \int_{0}^{1}\left(\mathcal{S}_{t} f+[\mathcal{S}, \mathcal{A}] f, f\right) d t \leq \mathbb{E} \int_{0}^{1}\left\|e^{\varphi} F+\widetilde{V} f\right\|^{2} d t-\mathbb{E} \int_{0}^{1}(\mathcal{S}(\widetilde{G} f), \widetilde{G} f) d t
$$

Next, from the definition of $\mathcal{S}$ we have $\mathcal{S}(\widetilde{G} f)=\widetilde{G} \mathcal{S} f+\Delta \widetilde{G} f+2 \nabla \widetilde{G} \cdot \nabla f$ and we observe that

$$
\int_{\mathbb{R}^{n}} \Delta \widetilde{G} \widetilde{G}|f|^{2} d x+2 \int_{\mathbb{R}^{n}} \nabla \widetilde{G} \cdot \nabla f \widetilde{G} f d x=-\int_{\mathbb{R}^{n}}|\nabla \widetilde{G}|^{2}|f|^{2} d x
$$


The latter implies that

$$
\begin{aligned}
& \mathbb{E} \int_{0}^{1}\|\mathcal{S} f\|^{2} d t+ \mathbb{E} \int_{0}^{1}\left(\mathcal{S}_{t} f+[\mathcal{S}, \mathcal{A}] f, f\right) d t \\
& \leq \mathbb{E} \int_{0}^{1}\left\|e^{\varphi} F+\widetilde{V} f\right\|^{2} d t+\mathbb{E} \int_{0}^{1}\left(\mathcal{S} f, f \widetilde{G}^{2}\right) d t+\mathbb{E} \int_{0}^{1}\|f \nabla \widetilde{G}\|^{2} d t \\
& \leq 2 \mathbb{E} \int_{0}^{1}\left\|e^{\varphi} F\right\|^{2} d t+c\|\widetilde{V}\|_{\infty}^{2} \mathbb{E} \int_{0}^{1}\|f\|^{2} \\
&+\frac{1}{2} \mathbb{E} \int_{0}^{1}\|\mathcal{S} f\|^{2}+\frac{1}{2}\|\widetilde{G}\|_{\infty}^{4} \mathbb{E} \int_{0}^{1}\|f\|^{2} d t+\|\nabla \widetilde{G}\|_{\infty}^{2} \mathbb{E} \int_{0}^{1}\|f\|^{2} d t
\end{aligned}
$$

Thanks to the definition of the space-time commutator $\mathcal{S}_{t}+[\mathcal{S}, \mathcal{A}]$ we conclude

$$
\frac{\epsilon R^{2}}{8 \mu} \mathbb{E} \int_{0}^{1}\|f\|^{2} d t \leq\left(2+c\|\widetilde{V}\|_{\infty}^{2}+\frac{1}{2}\|\widetilde{G}\|_{\infty}^{4}+\|\nabla \widetilde{G}\|_{\infty}^{2}\right) \mathbb{E} \int_{0}^{1}\|f\|^{2} d t+2 \mathbb{E} \int_{0}^{1}\left\|e^{\varphi} F\right\|^{2} d t
$$

Finally, we take $R>0$ large enough depending on $\widetilde{V}, \widetilde{G}, \nabla \widetilde{G}$ so that we can absorb the first term of the right-hand side in a fraction of the left-hand side. Hence

$\frac{\epsilon R^{2}}{16 \mu} \mathbb{E} \int_{0}^{1}\|f\|^{2} d t \leq \mathbb{E} \int_{0}^{1}\left\|e^{\varphi} F\right\|^{2} d t \leq 2 \mathbb{E} \int_{0}^{1}\left\|e^{\varphi} \theta_{M} \eta_{R}^{\prime} \widetilde{u}\right\|^{2} d t+2 \mathbb{E} \int_{0}^{1}\left\|e^{\varphi}\left(2 \nabla \theta_{M} \cdot \nabla \widetilde{u}+\Delta \theta_{M} \widetilde{u}\right) \eta_{R}\right\|^{2} d t$

We will study separately the two terms of the right-hand side. The first term is supported in $\left\{(x, t)|x| \leq 2 M, t \in\left[\frac{1}{2 R}, \frac{1}{R}\right] \cup\left[1-\frac{1}{R}, 1-\frac{1}{2 R}\right]\right\}$, where

$\mu\left|x+R t(1-t) e_{1}\right|^{2} \leq \mu(1+\epsilon)|x|^{2}+\mu\left(1+\frac{1}{\epsilon}\right) R^{2} t^{2}(1-t)^{2} \leq \mu(1+\epsilon)|x|^{2}+\mu\left(1+\frac{1}{\epsilon}\right) \leq \gamma|x|^{2}+\frac{\gamma}{\epsilon}$

Thus,

$$
\mathbb{E} \int_{0}^{1}\left\|e^{\varphi} \theta_{M} \eta_{R}^{\prime} \widetilde{u}\right\|^{2} d t \leq R^{2} e^{2 \frac{\gamma}{\epsilon}+\frac{R}{3}} \mathbb{E} \int_{0}^{1}\left\|e^{\gamma|x|^{2}} \widetilde{u}\right\|^{2} d t
$$

and Lemma 3.4 implies that the last integral is bounded by the assumptions on the solution.

Since the second term is supported in $x \in B_{2 M} \backslash B_{M}, t \in\left[\frac{1}{2 R}, 1-\frac{1}{R}\right]$, we notice that

$\mu\left|x+R t(1-t) e_{1}\right|^{2} \leq \mu(1+\epsilon)|x|^{2}+\mu\left(1+\frac{1}{\epsilon}\right) R^{2} t^{2}(1-t)^{2} \leq \mu(1+\epsilon)|x|^{2}+\mu\left(1+\frac{1}{\epsilon}\right) R^{2} \leq \gamma|x|^{2}+\frac{\gamma R^{2}}{\epsilon}$

Therefore, the contribution of the second term is bounded by

$$
\mathbb{E} \int_{0}^{1}\left\|e^{\varphi}\left(2 \nabla \theta_{M} \cdot \nabla \widetilde{u}+\Delta \theta_{M} \widetilde{u}\right) \eta_{R}\right\|^{2} d t \leq \frac{1}{M^{2}} e^{\frac{2 \gamma R^{2}}{\epsilon}+\frac{R^{2}}{3}} \mathbb{E} \int_{1 / 2 R}^{1-1 / 2 R}\left\|e^{\gamma|x|^{2}}(|\widetilde{u}|+|\nabla \widetilde{u}|)\right\|^{2} d t
$$


Moreover, if $t \in\left[\frac{1}{2 R}, 1-\frac{1}{2 R}\right], 1 \leq 4 R^{2} t(1-t)$ so

$$
\mathbb{E} \int_{1 / 2 R}^{1-1 / 2 R}\left\|e^{\gamma|x|^{2}}(|\widetilde{u}|+|\nabla \widetilde{u}|)\right\|^{2} d t \leq 4 R^{2} \mathbb{E} \int_{0}^{1}\left\|e^{\gamma|x|^{2}} t(1-t)(|\widetilde{u}|+|\nabla \widetilde{u}|)\right\|^{2} d t .
$$

Again, Lemma 3.4 shows that the last integral is bounded by the assumptions on the solution. Notice also that we conclude that the second term in 4.11 tends to zero when $M$ tends to zero.

On the other hand, if $(x, t) \in B_{\epsilon(1-\epsilon) R / 4} \times\left[\frac{1-\epsilon}{2}, \frac{1}{2}\right], \theta_{M}(x)=\eta_{R}(t)=1$ (i.e $\left.f=e^{\varphi} \widetilde{u}\right)$ and

$$
\varphi(x, t) \geq \frac{\mu R^{2}(1-\epsilon)^{2}}{16}-\frac{(1+\epsilon) R^{2}}{64 \mu}=\frac{R^{2}}{64 \mu}\left(4 \mu^{2}(1-\epsilon)^{2}-(1+\epsilon)\right)>0
$$

Altogether, we show that there exists $N>0$ depending on the decay hypothesis of the solution, $\gamma$ and $\epsilon$ (but not on $R$ ) such that

$$
\frac{\epsilon R^{2}}{16 \mu} e^{\frac{R^{2}}{32 \mu}\left(4 \mu^{2}(1-\epsilon)^{2}-(1+\epsilon)\right)} \mathbb{E}\|\widetilde{u}\|_{L^{2}\left(B_{\epsilon(1-\epsilon) R / 4} \times\left[\frac{1-\epsilon}{2}, \frac{\epsilon}{2}\right]\right)}^{2} \leq R^{2} e^{R / 3} N .
$$

By letting $R \rightarrow \infty$ we see that

$$
\mathbb{E}\|\widetilde{u}\|_{L^{2}\left(\mathbb{R}^{n} \times\left[\frac{1-\epsilon}{2}, \frac{\epsilon}{2}\right]\right)}^{2}=0 \Rightarrow \widetilde{u} \equiv u \equiv 0 \mathbb{P}-\text { a.s. }
$$

\section{ACKNowledgement}

This material is based upon work supported by the National Science Foundation under Grant No. DMS-1440140 while both authors were in residence at the Mathematical Sciences Research Institute in Berkeley, California, during Fall 2015 semester. The first author is partially supported by the projects ERCEA Advanced Grant 669689-HADE, MTM2014-53145-P and IT641-13. Both authors would like to thank L. Escauriaza and L. Vega for fruitful conversations. The authors would also like to thank C. Mueller and A. Debussche for discussions on stochastic conformal transformation, and thank S. Lototsky and F. Flandoli for discussions on interior regularity for stochastic PDEs.

\section{REFERENCES}

[1] Viorel Barbu, Aurel Răşcanu, and Gianmario Tessitore. Carleman estimates and controllability of linear stochastic heat equations. Applied Mathematics \& Optimization, 47(2):97-120, 2003.

[2] Juan Antonio Barcelo, Luca Fanelli, Susana Gutierrez, Alberto Ruiz, and Mari Cruz Vilela. Hardy uncertainty principle and unique continuation properties of covariant Schrödinger flows. Journal of Functional Analysis, 264(10):2386-2415, 2013.

[3] Aingeru Fernández Bertolin and Luis Vega. Uniqueness properties for discrete equations and carleman estimates. Journal of Functional Analysis, 272(11):4853-4869, 2017.

[4] Biagio Cassano and Luca Fanelli. Sharp hardy uncertainty principle and gaussian profiles of covariant Schrödinger evolutions. Transactions of the American Mathematical Society, 367(3):2213-2233, 2015.

[5] M Cowling, L Escauriaza, CE Kenig, G Ponce, and L Vega. The hardy uncertainty principle revisited. Indiana University Mathematics Journal, pages 2007-2025, 2010. 
[6] Miguel De Guzman. Differentiation of integrals in rn. In Measure theory, pages 181-185. Springer, 1976.

[7] L Escauriaza, CE Kenig, G Ponce, and L Vega. Hardy uncertainty principle, convexity and parabolic evolutions. Communications in Mathematical Physics, 346(2):667-678, 2016.

[8] Luis Escauriaza, C Kenig, Gustavo Ponce, and Luis Vega. Uniqueness properties of solutions to Schrödinger equations. Bulletin of the American Mathematical Society, 49(3):415-442, 2012.

[9] Luis Escauriaza, Carlos E Kenig, Gustavo Ponce, and L Vega. On uniqueness properties of solutions of Schrödinger equations. Communications in Partial Differential Equations, 31(12):1811-1823, 2006.

[10] Luis Escauriaza, Carlos E Kenig, Gustavo Ponce, and Luis Vega. Hardy's uncertainty principle, convexity and Schrödinger evolutions. Journal of the European Mathematical Society, 10(4):883-907, 2008.

[11] Luis Escauriaza, Carlos E Kenig, Gustavo Ponce, and Luis Vega. Uncertainty principle of morgan type and Schrödinger evolutions. Journal of the London Mathematical Society, 83(1):187-207, 2010.

[12] Luis Escauriaza, Carlos E Kenig, Gustavo Ponce, Luis Vega, et al. The sharp hardy uncertainty principle for Schrödinger evolutions. Duke Mathematical Journal, 155(1):163-187, 2010.

[13] Aingeru Fernández-Bertolin. Convexity properties of discrete Schrödinger evolutions and hardy's uncertainty principle. arXiv preprint arXiv:1506.03717, 2015.

[14] Franco Flandoli. Regularity Theory and Stochastic Flows for Parabolic SPDES, volume 9. CRC Press, 1995.

[15] Peng Gao, Mo Chen, and Yong Li. Observability estimates and null controllability for forward and backward linear stochastic kuramoto-sivashinsky equations. SIAM Journal on Control and Optimization, 53(1):475-500, 2015.

[16] GH Hardy. A theorem concerning fourier transforms. Journal of the London Mathematical Society, 1(3):227-231, 1933.

[17] Philippe Jaming, Yurii Lyubarskii, Eugenia Malinnikova, and Karl-Mikael Perfekt. Uniqueness for discrete Schrödinger evolutions. arXiv preprint arXiv:1505.05398, 2015.

[18] Qi Lü. Carleman estimate for stochastic parabolic equations and inverse stochastic parabolic problems. Inverse Problems, 28(4):045008, 2012.

[19] Qi Lu. Observability estimate for stochastic schrödinger equations and its applications. SIAM Journal on Control and Optimization, 51(1):121-144, 2013.

[20] Qi Lü and Zhongqi Yin. Unique continuation for stochastic heat equations. ESAIM: Control, Optimisation and Calculus of Variations, 21(2):378-398, 2015.

[21] Qi Lü and Xu Zhang. Global uniqueness for an inverse stochastic hyperbolic problem with three unknowns. Communications on Pure and Applied Mathematics, 68(6):948-963, 2015.

[22] Tu A. Nguyen. On a question of landis and oleinik. Trans. Amer. Math. Soc., 362(6):2875-2899, 2010.

[23] Daniel Revuz and Marc Yor. Continuous martingales and Brownian motion, volume 293. Springer Science \& Business Media, 2013.

[24] A Sitaram, M Sundari, and S Thangavelu. Uncertainty principles on certain lie groups. Proc. Indian Acad. Sci.(Math. Sci.), 105(2):135-151, 1995.

[25] Elias M Stein and Rami Shakarchi. Complex analysis. princeton lectures in analysis, ii, 2003.

[26] Gengsheng Wang, Ming Wang, and Yubiao Zhang. Observability and unique continuation inequalities for the Schrödinger equation. arXiv preprint arXiv:1606.05861, 2016.

[27] Donghui Yang and Jie Zhong. Observability inequality of backward stochastic heat equations for measurable sets and its applications. SIAM Journal on Control and Optimization, 54(3):1157-1175, 2016.

[28] Ganghua Yuan. Conditional stability in determination of initial data for stochastic parabolic equations. Inverse Problems, 33(3):035014, 2017.

[29] Xu Zhang. Carleman and observability estimates for stochastic wave equations. SIAM Journal on Mathematical Analysis, 40(2):851-868, 2008.

[30] Xu Zhang. Unique continuation for stochastic parabolic equations. Differential and Integral Equations, 21(12):81-93, 2008.

[31] Enrique Zuazua. Controllability and observability of partial differential equations: some results and open problems. Handbook of differential equations: evolutionary equations, 3:527-621, 2007. 